Relations industrielles

Industrial Relations

\title{
R. Blanpain (dir.), Comparative Labour Law and Industrial Relations in Industrialised Market Economies
}

\section{Pierre Verge}

Volume 46, numéro 4, 1991

URI : https://id.erudit.org/iderudit/050723ar

DOI : https://doi.org/10.7202/050723ar

Aller au sommaire du numéro

Éditeur(s)

Département des relations industrielles de l'Université Laval

ISSN

0034-379X (imprimé)

1703-8138 (numérique)

Découvrir la revue

Citer ce compte rendu

Verge, P. (1991). Compte rendu de [R. Blanpain (dir.), Comparative Labour Law and Industrial Relations in Industrialised Market Economies]. Relations industrielles / Industrial Relations, 46(4), 864-865.

https://doi.org/10.7202/050723ar

Tous droits réservés (C) Département des relations industrielles de l'Université Laval, 1991
Ce document est protégé par la loi sur le droit d'auteur. L’utilisation des services d'Érudit (y compris la reproduction) est assujettie à sa politique d'utilisation que vous pouvez consulter en ligne.

https://apropos.erudit.org/fr/usagers/politique-dutilisation/ 


\section{RECENSIONS BOOK REVIEWS}

Comparative Labour Law and Industrial Relations in Industrialised Market Economies, publié sous la direction de R. Blanpain, $4^{\mathrm{e}}$ édition, Deventer, Kluwer Law and Taxation Publishers, 1990, ISBN 906544666 (vol. 1) et ISBN 9065444955 (vol. 2)

Huit ans séparent cette quatrième édition révisée de l'ouvrage initial (recension dans Relations industrielles vol. 39, no 1, 1984, 201). Le professeur Blanpain réitère l'objectif dans sa préface: faire ressortir les principaux traits de la réalité évolutive du droit et des rapports du travail contemporains par des études thématiques qui intègrent et comparent, sous la plume de différents auteurs, les éléments d'un bon nombre d'ensembles nationaux et internationaux. Ceux-ci composent, dit-on cependant cette fois, le subunivers des économies de marché industrialisées. Comment et jusqu'à quel point la démarche a-t-elle évolué?

D'abord, l'enveloppe s'est modifiée: deux tomes regroupent désormais, l'un, treize études qualifiées de «droit de travail» - en plus de deux textes introductifs traitant respectivement de la problématique générale (Blanpain) et de la documentation (Vranken) - l'autre, dix études dites de «relations industrielles». La façon la plus utile de refléter la diversité de tous ces thèmes consiste sans doute à les décliner simplement selon leur ordre de présentation. Les textes du premier tome: catégories de travailleurs et contrats de travail (Barbagelata); nouveau travail (The New Labour Force, de Veneziani); égalité et interdiction de discrimination dans l'emploi (Ben Israel); détermination du salaire - approche institutionnelle (Eyraud); temps de travail (Thurman); flexibilité et sécurité d'emploi (Hepple); modes de représentation des employés sur les lieux du travail (Biagi); règlement des conflits de droit (Aaron); droit de la grève et du lock-out (Birk); droit communautaire européen (Hepple); droit international du travail (Valticos et Samson); normes de conduite (guidelines) pour les multinationales (Blanpain); conflits de lois (d.i.p.) relatifs au contrat de travail et aux relations industrielles (Gamillscheg). Ceux du second: les relations industrielles dans un ordre économique mondial en mutation (Clarke); liberté d'association (Creighton); mouvements syndicaux nationaux (Cella et Treu) et internationaux (Windmuller); organisations patronales (Oechslin); gestion des ressources humaines (Dowling et Schuler); négociation collective (Cordova); perspectives et tendances en matière de conflits du travail (Clarke); règlement des conflits d'intérêts (Goldman) et, enfin, les relations du travail dans le secteur public (Ozaki). Des thèmes nouveaux sont donc venus s'ajouter: outre ce dernier, ceux relatifs à la transformation du travail, au salaire et au temps de travail, à la gestion des ressources humaines, de même qu'aux conflits de lois. De plus, cinq autres chapitres ont été confiés à de nouveaux auteurs (ceux relatifs à la documentation, au droit européen, à la discrimination, à la représentation des travailleurs - du moins, s'il y a correspondance avec l'ancien chapitre traitant de la participation, qui était de portée plus restreinte - et celui ayant trait à la liberté syndicale).

Cette nouvelle répartition formelle des thèmes conduit à des résultats discutables. Si l'on accepte bien que la négociation collective, y compris les rapports du travail dans la fonction publique, malgré ses aspects juridiques, fasse partie du domaine plus large des «relations industrielles», en revanche, il est difficile de ne pas voir dans la liberté d'association, du moins comme on en traite ici, un aspect de l'ordre juridique. Et même, une vision juridique pluraliste 
ne porte-t-elle pas naturellement sur la négociation collective? Par ailleurs, la détermination salariale pourrait assez bien suivre la négociation collective et l'évolution des modes de travail est un sujet tout aussi ambivalent... Au-delà de ces difficultés en partie formelles, la nouvelle répartition de la matière signifie aussi l'abandon de celle que l'on proposait initialement entre les thèmes à dimension internationale et les études proprement comparatives. Or, celle-ci correspondait à une disparité substantielle qui demeure bien présente dans les présentes études.

Certains textes, en effet, décrivent des phénomènes qui transcendent les frontières nationales: les associations syndicales internationales, ou encore, sur le plan juridique la présentation des normes internationales du travail et de leur contexte institutionnel, les règles des conflits de lois, ou encore, les lignes de conduite à l'intention des multinationales. Ils s'intercalent cependant entre d'autres contributions qui traduisent, avec toute l'intensité voulue, la démarche comparatiste. À titre illustratif, il en est ainsi de la préoccupation relative à l'étendue de la protection du travailleur face à la mutation du lien d'emploi que l'on retrouve chez Barbagelata et Veneziani, ou encore, advenant des réductions de personnel, chez Hepple. La détermination des frontières du droit de grève (Birk), celles du domaine de l'action syndicale (Cella et Treu), les niveaux, les sujets et les modes de négociation collective (Cordova), les modèles de représentation collective dans l'entreprise (Biagi) et ceux de règlement des conflits de droit (Aaron) conduisent aussi à une confrontation serrée des solutions nationales. La liberté d'association (Creighton), par contre, ne s'appréhende ici qu'à travers le prisme des grands instruments internationaux - si importants soient-ils eux-mêmes par ailleurs - sans se laisser voir vivre dans les sociétés plus ou moins libérales industrialisées.

Tout en laissant subsister une section «internationale» bien identifiée, la démarche comparatiste, qui correspond au titre de l'ouvrage, devrait même aller s'accentuant au fil des éditions ultérieures. Une voie tend à la confrontation de plus en plus intégrale des ensembles comparés, dans leurs dimensions à la fois socio-économiques, institutionnelles et plus strictement juridiques. Par exemple, pour ce qui est de ces dernières, elles pourront conduire à tenir compte, pourvu que l'objet de la comparaison y conduise naturellement, de l'état des libertés civiles dans les milieux comparés, ou encore, de l'intensité de la notion générale d'ordre public qui y prévaut généralement. Une restriction du champ territorial de la comparaison paraît alors inévitable.

Une autre avenue - celle que semblent privilégier la majorité des auteurs ici réunis - est celle, plus généralisante, de la comparaison systémique. Elle peut conduire, selon les cas, à des analyses axées sur des types de structures ou de fonctions. L'étude de Biagi portant sur les formes de représentation des employés sur les lieux de travail en est une excellente illustration. Par rapport à cette seconde approche, l'aire des économies de marché avancées, limite que ne s'imposait pas l'ouvrage initial, représente un défi audacieux, mais réaliste. Des auteurs réussissent cependant parfois à s'en évader, ainsi Cella et Treu (p. 61) et Windmuller (p. 97).

Le présent ouvrage constitue sans doute présentement une des plus riches réunions de synthèses comparatives consacrées aux rapports du travail. Un même courant les traverse: l'adaptation des institutions et des règles aux impératifs de flexibilité du travail résultant de l'ordre économique actuel - assouplissement de règles relatives au travail atypique (Veneziani, p. 67), souplesse dans la détermination du salaire (Eyraud, p. 131), du temps de travail (Thurman, p. 167), du niveau de personnel dans l'entreprise (Hepple, p. 178), au sein des communautés européennes (Hepple, p. 303-305), etc. Les réponses à ce besoin varient partiellement encore d'une contrée à l'autre, mais, en plus longue portée, un processus d'uniformisation, d'《internationalisation», en fait, des règles du travail n'est-il pas déjà engagé?

Pierre Verge 